\title{
Observation of Nonlinear Coupling between Low Frequency Coherent Modes and Background Turbulence in LMD-U*)
}

\author{
Tatsuya KOBAYASHI, Shigeru INAGAKI ${ }^{1,2)}$, Hiroyuki ARAKAWA, Stella OLDENBÜRGER ${ }^{2)}$, \\ Makoto SASAKI $^{1,2)}$,Yoshihiko NAGASHIMA ${ }^{2,3)}$, Takuma YAMADA ${ }^{2,3)}$, Satoru SUGITA, \\ Masatoshi YAGI ${ }^{1,2)}$, Naohiro KASUYA ${ }^{2,4)}$, Akihide FUJISAWA ${ }^{1,2)}$, \\ Sanae-I. ITOH ${ }^{1,2)}$ and Kimitaka ITOH ${ }^{2,4)}$ \\ Interdisciplinary Graduate School of Engineering Sciences, Kyushu University, Fukuoka 816-8580, Japan \\ ${ }^{1)}$ Research Institute for Applied Mechanics, Kyushu University, Fukuoka 816-8580, Japan \\ 2) Itoh Research Center for Plasma Turbulence, Kyushu University, Fukuoka 816-8580, Japan \\ ${ }^{3)}$ Graduate School of Frontier Sciences, The University of Tokyo, Tokyo 277-8561, Japan \\ ${ }^{4)}$ National Institute for Fusion Science, Toki 509-5292, Japan
}

(Received 7 December 2010 / Accepted 10 May 2011)

\begin{abstract}
A solitary wave like fluctuating structure is observed in the Large Mirror Device-Upgrade (LMD-U) under relatively high neutral pressure conditions. Ion saturation current fluctuations are measured and Fourier and wavelet power spectra are calculated. Temporal behavior of fluctuation power and strength of nonlinear coupling of low frequency coherent mode and background turbulence in a specific frequency region is analyzed. This is achieved using wavelet spectral and bispectral analyses with conditional averaging.
\end{abstract}

(C) 2011 The Japan Society of Plasma Science and Nuclear Fusion Research

Keywords: linear plasma, drift wave, turbulence, bispectral analysis, wavelet analysis, nonlinear coupling

DOI: $10.1585 /$ pfr.6.2401082

\section{Introduction}

Understanding of turbulence structure formation in magnetized plasma is important for realizing thermonuclear fusion. Theoretical and experimental works report that meso-scale structures such as zonal flows and streamers, which play an important role for plasma confinement, can be excited through nonlinear couplings of drift waves [1-11]. The nonlinear couplings, especially three-wave coupling in stationary state are studied by use of the bispectral analysis, a third order correlation of Fourier components [12]. The bispectral analysis, which can detect the mutual correlation of three waves, however, cannot clarify the causal relation of the coupling. Analysis of plasma turbulence dynamics must be developed to demonstrate causal relations [13-16]. Recently, bispectral analysis could be applied for dynamic phenomena by employing wavelet component instead of Fourier component [15-17].

The low temperature linear cylindrical plasma in the Large Mirror Device-Upgrade (LMD-U) can feature many types of nonlinear fluctuations depending on the discharge conditions i.e. magnetic field strength $(B=0.03-0.15 \mathrm{~T})$ and filling neutral gas pressure ( $p_{n}=2-6$ mTorr). With medium magnetic field strength and relatively low neutral pressure, a streamer has been discovered using bispectral analysis in frequency and poloidal wave number

author'se-mail: kobayashi@ riam.kyushu-u.ac.jp

*) This article is based on the presentation at the 20th International Toki Conference (ITC20).

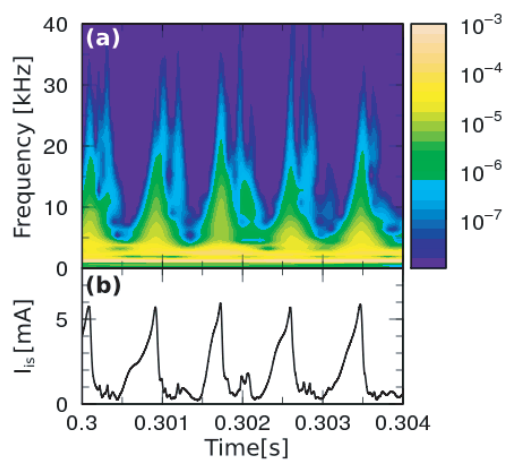

Fig. 1 (a) Wavelet power spectrum of normalized ion saturation current fluctuations $\tilde{I}_{\text {is }} / \bar{I}_{\text {is }}$ taken at one point of a multichannel probe array. (b) Time evolution of $\tilde{I}_{\text {is }}$.

space [18]. In different experimental conditions, a zonal flow has been observed and the corresponding energy flows could be clarified [19]. A low frequency and low poloidal mode number fluctuation has been identified as an important player for the generation of meso-scale structures.

Yet another type of nonlinear fluctuation is a solitary wave like fluctuation, that appears in higher neutral pressure condition ( $p_{n}=5 \mathrm{mTorr}$ ), and medium magnetic field strength $(B=0.09 \mathrm{~T})$ [20]. Figure 1 (a) shows the wavelet power spectrum of the ion saturation current fluctuation $\tilde{I}_{\text {is }}$ measured with one probe tip of a multi-channel Langmuir probe array [21] localized at $r=40 \mathrm{~mm}$, where the scale length of the electron density gradient becomes minimum (raw signal is also shown in Fig. 1 (b)). In this paper, the 
Morlet function with $\omega_{0}=6$ [22] is used as mother wavelet. The wavelet power spectrum is normalized $\left(\tilde{I}_{\text {is }} / \bar{I}_{\text {is }}\right)$ and used as an index of density fluctuations. The fluctuation components of higher frequencies $(f>5 \mathrm{kHz})$ dynamically appear and disappear, while lower frequency modes ( $f<5 \mathrm{kHz}$ ) always exist. This dynamic behavior of fluctuations is linked to the triangle wave like shape of the raw signal. The existence of mutual nonlinear couplings among low frequency coherent modes $(f<10 \mathrm{kHz})$ had been reported earlier [23]. In this article, the dynamic behavior of nonlinear couplings is analyzed by use of wavelet bispectral analysis.

\section{Analysis Method}

To allow nonlinear coupling among three waves $\Psi\left(f_{1}\right), \Psi\left(f_{2}\right)$, and $\Psi\left(f_{3}\right)$, the frequencies have to satisfy the matching condition $f_{3}=f_{1} \pm f_{2}$. Here $\Psi(f)$ indicates the Fourier component of an arbitrary physical quantity. Squared bicoherence $\hat{b}^{2}\left(f_{3}\right)$ defined as,

$$
\hat{b}^{2}\left(f_{3}\right)=\frac{\left|\left\langle\Psi\left(f_{1}\right) \Psi\left(f_{2}\right) \Psi^{*}\left(f_{3}\right)\right\rangle\right|^{2}}{\left\langle\left|\Psi\left(f_{1}\right) \Psi\left(f_{2}\right)\right|^{2}\right\rangle\left\langle\left|\Psi\left(f_{3}\right)\right|^{2}\right\rangle},
$$

expresses the degree of conservation of phase relation among three waves. The bracket and asterisk in Eq. (1) represent the ensemble average and the complex conjugate, respectively. Bicoherence $\hat{b}\left(f_{3}\right)$ becomes unity if the phase relation of three waves is completely conserved. A finite time window is required for the calculation of Fourier components, and a large number of ensemble averages is necessary to obtain a significant bicoherence [24]. The dynamics of nonlinear coupling are difficult to measure by use of such a bicoherence technique. By employing the wavelet components $W(f)$ instead of Fourier components, it is possible to define the so-called wavelet bicoherence. A conditional averaging is then applied to the decomposed data in order to clarify the temporal characteristics of nonlinear coupling. The criterion for data selection is the phase of the target mode. The fluctuation period of the target mode is divided into regular intervals, $\theta_{1}, \theta_{2}, \cdots, \theta_{i}, \cdots, \theta_{n}$, where we define $\theta_{i}=-\pi+2(i-1) \pi / n$, and the squared wavelet bicoherence for the $i$ 's segment $\hat{b}_{W, i}^{2}\left(f_{3}\right)$

$$
\hat{b}_{W, i}^{2}\left(f_{3}\right)=\left.\frac{\left|\left\langle W\left(f_{1}\right) W\left(f_{2}\right) W^{*}\left(f_{3}\right)\right\rangle\right|^{2}}{\left\langle\left|W\left(f_{1}\right) W\left(f_{2}\right)\right|^{2}\right\rangle\left\langle\left|W\left(f_{3}\right)\right|^{2}\right\rangle}\right|_{\theta_{i}<\theta<\theta_{i+1}} .
$$

If the wavelet bicoherence $\hat{b}_{W, i}\left(f_{3}\right)$ converges to a significant value, the temporal evolution of nonlinear coupling strength can be discussed.

\section{Experimental Setup and Basic In- formation on Solitary Wave}

The cylindrical stainless steel vacuum vessel of the LMD-U with a length of $3740 \mathrm{~mm}$ and a diameter of $450 \mathrm{~mm}$ is surrounded by Helmholtz coils which can produce a homogeneous magnetic field parallel to the axis of

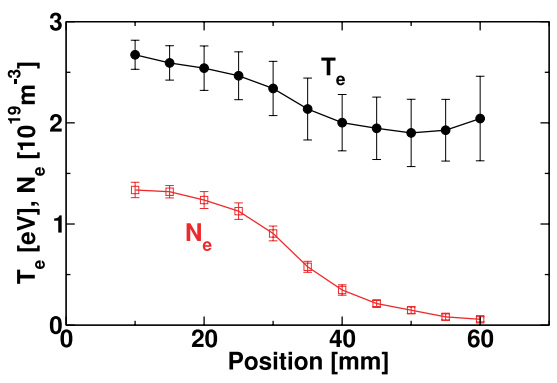

Fig. 2 Static radial profile of electron temperature and electron density measured with a double probe (sweep frequency $f_{\mathrm{sw}}=100 \mathrm{~Hz}$ ). Error bars correspond to the standard deviation of each fitting.

the vacuum vessel. A double loop antenna producing the RF helicon Argon plasma discharge $(3 \mathrm{~kW}, 7 \mathrm{MHz})$ is installed on a quartz tube with a length of $400 \mathrm{~mm}$ and a diameter of $100 \mathrm{~mm}$ at one side of the vessel (a schematic is shown in ref [23]). The typical value of electron temperature and electron density in LMD- $\mathrm{U}$ is $2-3 \mathrm{eV}$ and $1 \times 10^{-19} \mathrm{~m}^{-3}$ respectively. Figure 2 shows the static radial profile of electron temperature and electron density. The gradient of the density profile becomes shorter around $r=40 \mathrm{~mm}$ where the multi-channel probe array is mounted. In the high neutral pressure case, frequent ionneutral collision damp collisional drift wave [25] and only a few linear unstable modes are allowed to grow.

Figure 3 shows the static characteristics of plasma fluctuations. Figure 3 (a) shows the power spectrum of normalized ion saturation current fluctuation $\tilde{I}_{\text {is }} / \bar{I}_{\text {is }}$ on one probe tip, Fig. 3 (b) shows the total squared bicoherence calculated by summation of the squared bicoherence along $f_{3}$ constant lines. The two dimensional power spectrum in frequency and poloidal mode number space is also calculated simultaneously from 32 points of the multi-channel probe array. Identified mode numbers of each peak are labeled in Fig. 3(a). A significantly strong coherent mode at $f=1.2 \mathrm{kHz}, m=1$ (fundamental mode) is observed, besides higher harmonics up to $m=10$. The results of spectral analyses (power spectrum, cross-coherence and cross-phase) of the $m=1$ ion saturation current fluctuations and the $m=1$ floating potential fluctuations are shown in [23]. The $m=1$ mode has an amplitude ratio of $\sim 0.6$, a cross-coherence of $\sim 1$ and a cross-phase of $\sim \pi / 6$ between ion saturation current and floating potential, and is presumed to be a linearly unstable resistive drift wave. The power of the turbulent fluctuations in the frequency of $f>20 \mathrm{kHz}$ are proportional to $f^{-5.5}$. It has been confirmed that the correlation length of background turbulence in this frequency region is much shorter than that of the coherent mode. The bicoherence of turbulence in the frequency of $f>20 \mathrm{kHz}$ is not strong but its spectrum is much wider than for the coherent modes. The total squared bicoherence is sensitive to this fact. A lattice pattern of strong couplings is seen in the low frequency region in Fig. 3 (d). This is because modes in the low frequency region strongly 

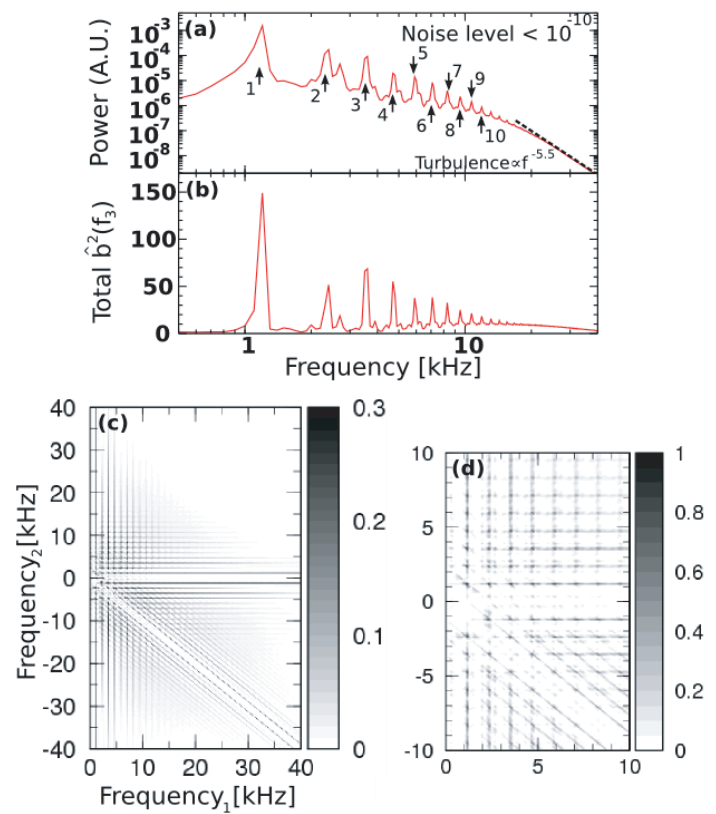

Fig. 3 (a) Power spectrum of normalized ion saturation current $\tilde{I}_{\text {is }} / \bar{I}_{\text {is }}$ from a one point measurement of the multi-channel probe array. (b) Total squared bicoherence along $f_{3}$ constant lines. (c) Squared bicoherence of ion saturation current fluctuations. (d) Extended figure of (c). A time window of $10 \mathrm{~ms}$ is used for the FFT and 720 ensemble averages are performed for (a). For (b) and (c), 20 probes are used to check the convergence of bicoherence. The numbers on (a) express the poloidal mode number of each peak calculated using the multi-channel probe array.

couple with the fundamental mode and all higher harmonics. When the solitary wave is excited, such as, in LMD-U, such a mutual coupling is one of the characteristic features. In addition, turbulence in the frequency of $f>20 \mathrm{kHz}$ also couples with low frequency modes.

\section{Wavelet Bispectral Analysis}

Figure 1 indicates that higher harmonics gain and lose energy with the cycle of the $m=1$ mode. The conditional average technique decomposing this cycle is applied to the calculation of power spectrum and wavelet bicoherence. The phase of the $m=1$ mode is divided in four segments and is used for the classification of the wavelet components, after the usual wavelet decomposition. Typical splitting of the raw data is shown in Fig. 4(a). The wavelet components out of the cone of influence [22] are rejected. Figure 4 (b) shows the variation of the power spectrum. When the $m=1$ mode is in the phase $-0.5<$ $\theta / \pi<0$, the fluctuation power of lower frequency coherent modes increases along with the decrease of the fluctuation power of background turbulence in the frequency region $f>20 \mathrm{kHz}$. The fluctuation powers of both coherent mode and this turbulence become maximum when the phase is $0<\theta / \pi<0.5$. Figures 4 (c)-(f) show the temporal variation of squared bicoherence. Different features of nonlinear couplings are found in the four phases. The non-
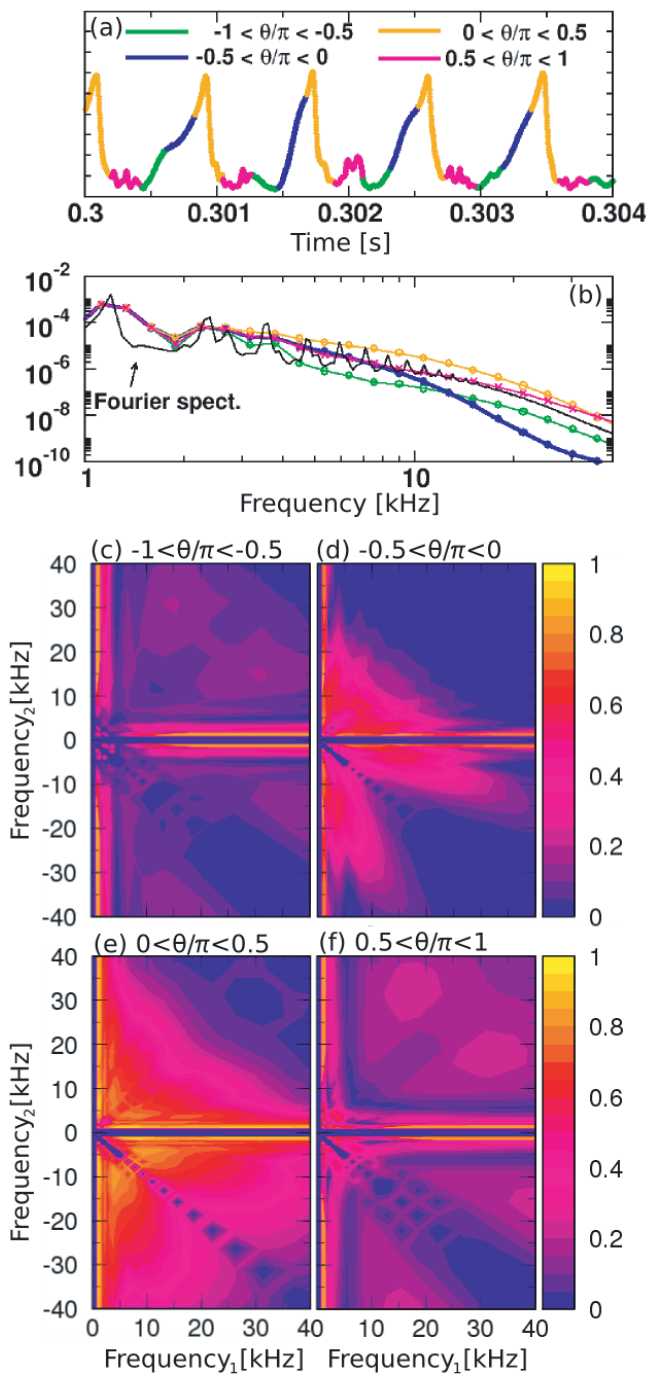

Fig. 4 Results of conditional averaged wavelet analysis. (a) The typical time evolution of raw data and the splitting of the data, (b) averaged power spectra in each phase, (c)-(f) squared wavelet bicoherence.

linear couplings of coherent modes are most active when the $m=1$ mode is in the phase $0<\theta / \pi<0.5$, that is the time just after the peak of $m=1$ mode. On the other hand, strength of nonlinear coupling of turbulence in the frequency region of $f>20 \mathrm{kHz}$ decreases when that mode is in the phase $-0.5<\theta / \pi<0$, and becomes strongest in the phase $0.5<\theta / \pi<1$.

The time evolution of nonlinear couplings of some specific modes is shown in more detail. We categorize the temporal features of nonlinear coupling in two types using the ratio of maximum and minimum value of squared bicoherence. A bicoherence with only slight variation (the ratio of maximum and minimum $\hat{b}^{2}\left(f_{3}\right)$ is less than 0.5$)$ for a specific component combination is called stationary coupling. In the opposite case we use the term dynamic coupling. Figures 5 (a) and (b) show the typical time evolutions of squared bicoherence categorized in stationary and dynamic coupling, respectively. In Fig. 5 (a), the strength of $(1,1,2)$ coupling (black curve) does not 


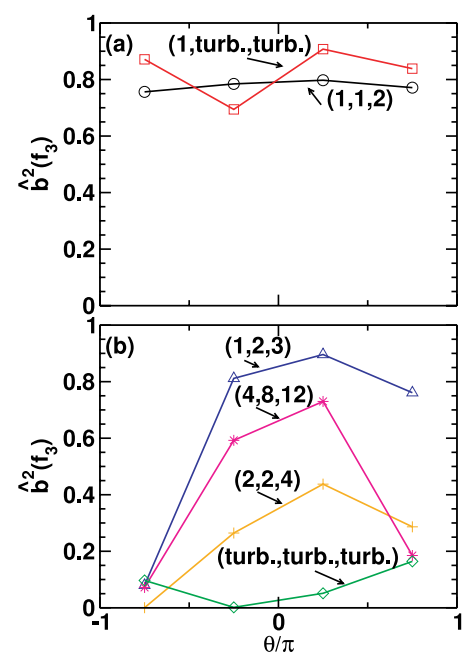

Fig. 5 Time evolutions of squared bicoherence categorized in (a) stationary coupling and (b) dynamic coupling. Each curve is identified by labels, which indicate the fluctuation modes $\left(\tilde{I}_{\text {is }}\left(f_{1}\right), \tilde{I}_{\text {is }}\left(f_{2}\right), \tilde{I}_{\text {is }}\left(f_{3}=f_{1}+f_{2}\right)\right)$ using the numbers in Fig. 3 (a). Squared bicoherences concerning turbulence ((1,turb.,turb.) and (turb.,turb.,turb.)) are calculated using specific frequency components in the frequency region of $f>20 \mathrm{kHz}((1.2 \mathrm{kHz}, 25.0 \mathrm{kHz}$, $26.2 \mathrm{kHz})$ and $(25.0 \mathrm{kHz}, 25.0 \mathrm{kHz}, 50.0 \mathrm{kHz})$, respectively).

change in any phase. That of (1,turbulence,turbulence) coupling (red curve) is also stationary, expect for the phase $-0.5<\theta / \pi<0$. In Fig. 5 (b), squared bicoherence of $(1,2,3),(4,8,12)$ and $(2,2,4)$ varies much temporally, and becomes maximum when $0<\theta / \pi<0.5$. It can be noticed that the rough frequency resolution of wavelet spectrum cannot distinguish the temporal behavior of each peak, but expresses the general temporal feature of harmonics. In the laboratory frame, higher harmonics are considered to be quasi-mode because their strength of nonlinear coupling as well as fluctuation power becomes maximum after the growth of the $m=1$ mode. On the other hand, the strength of three modes nonlinear couplings in the turbulence region of frequencies decreases just before the $m=1$ mode reaches its peak value.

\section{Discussion and Summary}

From the dynamic analysis of nonlinear coupling, the temporal sequence of mode excitation in the laboratory frame is deduced as following. First, the $m=1$ mode is excited by linear processes. Second, the $m=1$ mode feeds the energy to higher harmonics by significantly strong three-wave couplings when the value of the $m=1$ ion saturation current fluctuation becomes maximum, and further harmonic modes are excited gradually. The fluctuation power and strength of nonlinear couplings of the background turbulence decrease. The strong coupling no longer continues and decays when the value of the $m=1$ ion saturation current fluctuation reaches the minimum. Finally, the power of the harmonics decreases and the strength of nonlinear coupling among background turbulence increases. In addition, the linearly excited $m=1$ mode nonlinearly permanently couples with the turbulence. There is an obvious phase delay between mode excitation and nonlinear coupling processes between the low frequency coherent mode and the background turbulence in the frequency region of $f>20 \mathrm{kHz}$. This type of temporal behavior is not observed in other experimental condition e.g. in low neutral pressure conditions. A detailed comparison of temporal behavior of nonlinear couplings and their behavior in the plasma frame is left for future work.

In this article, time evolutions of fluctuation power and nonlinear coupling of low frequency coherent modes and background turbulence in the specific frequency region of $f>20 \mathrm{kHz}$ are observed by using wavelet spectral and bispectral analyses with conditional averaging. Experimental observations indicate that fluctuation power and nonlinear couplings change depending on the phase of the $m=1$ mode.

\section{Acknowledgment}

This work was partly supported by a Grant-in-Aid for Scientific Research (S) from the Japan Society for the Promotion of Science (21224014) and the collaboration programs of RIAM at Kyushu University and the National Institute of Fusion Science (NIFS07KOAP017).

[1] G. Tynan et al., Plasma Phys. Control. Fusion 51, 113001 (2009).

[2] A. Fujisawa, Nucl. Fusion 49, 013001 (2009).

[3] P.H. Diamond et al., Plasma Phys. Control. Fusion 47, R35 (2005).

[4] K. Itoh et al., Phys. Plasmas 13, 055502 (2007).

[5] M. Yagi et al., Plasma Phys. Control. Fusion 48, A409 (2006).

[6] M. Sasaki et al., Contrib. Plasma Phys. 48, No. 1-3, 68 (2008).

[7] Z. Lin et al., Science 281, 1835 (1998).

[8] A. Fujisawa et al., Phys. Rev. Lett. 93, 165002 (2004).

[9] Y. Nagashima et al., Phys. Rev. Lett. 95, 095002 (2005).

[10] N. Kasuya et al., Phys. Plasmas 15, 052302 (2008).

[11] P. Beyer et al., Phys. Rev. Lett. 85, 4892 (2000).

[12] G. Tynan et al., Phys. Plasmas 8, 2691 (2001).

[13] P. Manz et al., Plasma Phys. Control. Fusion 51, 035008 (2009).

[14] P. Manz et al., Phys. Rev. Lett. 103, 165004 (2009).

[15] A. Fujisawa et al., Phys. Rev. Lett. 98, 165001 (2007).

[16] A. Fujisawa et al., Plasma Phys. Control. Fusion 49, 211 (2007).

[17] F. Brochard et al., Phys. Plasmas 13, 122305 (2006).

[18] T. Yamada et al., Nature Phys. 4, 721 (2008).

[19] Y. Nagashima et al., Phys. Plasmas 16, 20706 (2009).

[20] T. Yamada et al., Plasma Fusion Res. 3, 44 (2008).

[21] T. Yamada et al., Rev. Sci. Instrum. 78, 123501 (2007).

[22] C. Torrence et al., Bull. Am. Meteorol. Soc. 79, 1, 61 (1998).

[23] T. Kobayashi et al., Plasma Fusion Res. 5, S2047 (2010).

[24] D. Grésillon et al., Phys. Fluids 31, 1904 (1988).

[25] M. Ignatenko et al., Jpn. J. Appl. Phys. Part1, 46, 1680 (2007). 